\title{
Matrix-Matrix Multiplications and Fault Tolerance on Hypercube Multiprocessors
}

\author{
Yuh-Rong Leu, Ing-Yi Chen* and Sy-Yen Kuo \\ Department of Electrical Engineering \\ National Taiwan University \\ *Department of Electronic Engineering \\ Taipei, Taiwan, R.O.C. \\ Chung Yuan Christian University \\ Chungli, Taiwan, R.O.C.
}

\begin{abstract}
Several new algorithms for matrix-matrix multiplications on hypercube multiprocessors are presented and evaluated based on the number of multiplications, additions, and transfers. The matrices to be multiplied are uniformly distributed to all processors of a hypercube system. Each processor owns some submatrices which are derived by dividing the source matrices. Each submatrix multiplication can now be performed independently within a processor. All the partial results are then summed up and transferred to a single processor. An orthogonal tree is used for efficient communication. The time complexity is $O\left(\log _{2} p\right)$ if $p \times p$ processors are used. In addition, the UDD (Uniform Data Distribution) approach is employed when some processors do not work properly and the faulty effects have been detected. Two classes of fault patterns are considered and evaluated.
\end{abstract}

\section{1: Basic Matrix-Vector Multiplication Algorithm}

The matrix-matrix multiplication algorithms presented in this paper are extensions of the matrix-vector multiplication algorithm in [1]. The basic matrix-vector multiplication can be described as the inner product of a vector with each row of a given matrix [3]:

$$
\begin{gathered}
\text { If } A \in R^{m \times n}, x \in R^{n}, \text { then } \\
y=A \cdot x, \text { where } y_{i}=\sum_{j=1}^{n} A_{i j} \cdot x_{j}, i=1, \ldots, m .
\end{gathered}
$$

Alternatives in viewing a matrix-matrix multiplication are listed below:

$$
\begin{aligned}
& \text { If } A \in R^{m \times n}, B \in R^{n \times q}, C \in R^{m \times q} \text {, then } \\
& \text { (1) } C=A \cdot B \text {, where } C_{i j}=\sum_{k=1}^{n} A_{i k} \cdot B_{k j} \\
& \text { (2) } C^{j}=A \cdot B^{j}
\end{aligned}
$$

In the above definitions, $A^{k}$ denotes the $k$-th column of $A$ and $A_{k}$ denotes the $k$-th row of $A$.

A common data mapping scheme for matrices is to use a two-dimensional grid embedding for the hypercube processors. To optimize the use of each processor, it is desirable to incorporate all nodes (processors) into the grid and distribute the matrix elements as evenly as possible among the nodes. A grid may be embedded on the hypercube using all nodes by numbering the processors according to the Gray code which is a binary number representation where only one bit is different for any two given consecutive numbers [4]. Fig. 1 illustrates such a Gray code numbering on a 5-D hypercube.

Acknowledgment: This research was supported by the National Science Council, Taiwan, R.O.C. , under Grant NSC 82-0404-E033-049-T and NSC 82-0408-E002-021. 


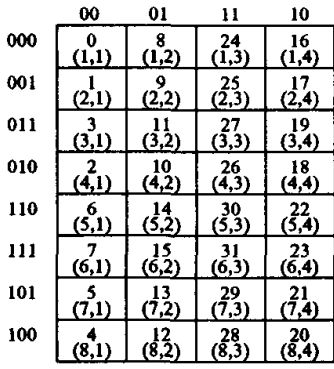

Fig. 1. Grid embedding.

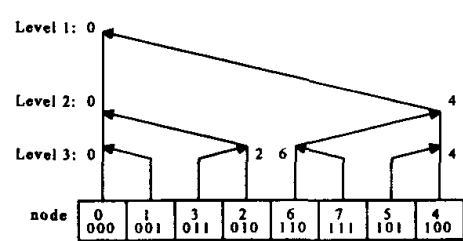

Fig. 2. Tree communication.

Suppose we need to sum up the values residing in the processors of column 1 in Fig. 1 . The steps taken are shown in Fig. 2, where $\rightarrow$ indicates the data-flow direction. As soon as a processor receives data, it sums up the data with its own data.

The basic matrix-vector multiplication algorithm can then be expressed as follows:

1. Distribute the matrix $A$ and the vector $x$ to each processor according to its position $(i, j)$ in the grid; thus, each processor acquires a portion of $A\left(A_{i j}\right)$ and a portion of $x\left(x_{j}\right)$.

2. Each processor computes sequentially the intermediate subvector $y_{i j}=A_{i j} \cdot x_{j}$.

3. Use the tree communication scheme row-wisely to gather and compute the resultant subvectors $Y$ distributed on the first column-processors. The equation below describes this operation:

$$
Y_{i}=\sum_{j=1}^{p} A_{i j} \cdot x_{j}=\sum_{j=1}^{p} y_{i j} .
$$

4. Collect all $Y_{i}$ 's into processor $(0,0)$ using the tree communication column-wisely.

\section{2: Matrix-Matrix Multiplication Algorithms}

The first algorithm is a simple approach. The distributing method of this algorithm is similar to the basic matrix-vector multiplication algorithm. In each computation cycle, only one column of matrix $B$ is processed. Matrix $A$ is distributed, and so each processor has a submatrix $A_{l y}$. Each $B^{j}$ is distributed across the processors of a row. $C^{j}$ is formed at the end of the $j$-th iteration. And in each iteration, a matrix-vector multiplication is performed as in the basic matrix-vector multiplication algorithm. So the entire matrix-matrix multiplication needs $q$ computation cycles (see Fig. 3(a))

The second one is named CE (Column-Expansion). In this algorithm, we use two augmented matrices each with $m$ rows and $n q$ columns. Each row of the augmented matrix consists of $q$ copies of a row of matrix $A$. Associated with each copy is a column of matrix $B$ (see Fig. 3(b)). This algorithm is based on the fact that $C_{i}=A_{i} \cdot B^{j}$. After each processor has completed its own tasks of multiplications, additions, and transfers, the entries of each row of matrix $C$ are distributed across the row-processors. So the tree communication scheme must be invoked to gather these data into the leftmost column-processors. In Fig. 3(b), $B^{\text {jt }}$ stands for the transposed vector of $B$ 's $j$-th column.

The third is the RE (Row-Expansion) algorithm. Algorithm RE still needs two augmented matrices - one formed by matrix $A$ and the other by $B$. But instead of expanding by the column, we expand the matrices by the row. Thus we have two matrices, each of size 
$m q \times n$. The entries of these two matrices are then evenly distributed to all the processors. The computational mechanism is similar to that of the Simple Algorithm, but only one computation cycle is required (see Fig. 3(c)). Fig. 4 gives the comparisons between previous and proposed algorithms.

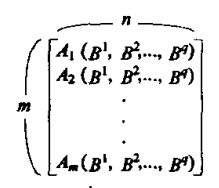

$q$ computation cycles

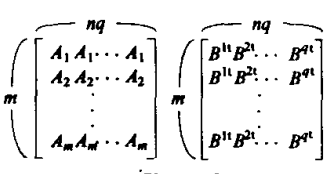

size $m \times n q$

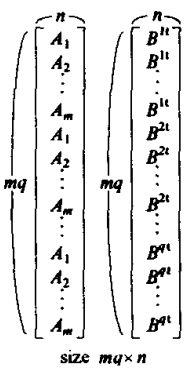

(c) RE

(a) Simple

(b) $\mathrm{CE}$

Fig. 3. Data distributions for matrix-matrix multiplications.

\begin{tabular}{|c|c|c|c|}
\hline & Multiplications & Additions & Transfers \\
\hline Sequential & $m \cdot q \cdot n$ & $m \cdot q \cdot(n-1)$ & 0 \\
\hline 2-D Grid & $q \cdot\left\lceil\frac{m}{p}\right\rceil \cdot\left\lceil\frac{n}{p}\right\rceil$ & $q \cdot\left\lceil\frac{m}{p}\right\rceil \cdot\left[\left(\left\lceil\frac{n}{p}\right\rceil-1\right)+p\right]$ & $q \cdot\left\lceil\frac{m}{p}\right\rceil \cdot p$ \\
\hline Simple Algorithm & $q \cdot\left\lceil\frac{m}{p}\right\rceil \cdot\left\lceil\frac{n}{p}\right\rceil$ & $q \cdot\left\lceil\frac{m}{p}\right\rceil \cdot\left[\left(\left\lceil\frac{m}{p}\right\rceil-1\right)+\log _{2} p\right]$ & $q \cdot\left\{\frac{m}{p}\right\rceil \cdot \log _{2} p$ \\
\hline Algorithm CE & $\left\lceil\frac{m}{p}\right\rceil \cdot\left\lceil\frac{n q}{p}\right\rceil$ & $\left.\left\lceil\frac{m}{p}\right\rceil \cdot\left(\frac{n q}{p}\right\rceil-1\right)$ & $\left\lceil\frac{m}{p}\right\rceil \cdot\left[\left\lceil\frac{q}{p}\right\rceil(p-1)+1\right]$ \\
\hline Algorithm RE & $\left\lceil\frac{m q}{p}\right\rceil \cdot\left\lceil\frac{n}{p}\right\rceil$ & $\left.\left\lceil\frac{m q}{p}\right\rceil \cdot\left[\left(\frac{n}{p}\right]-1\right)+\log _{2} p\right]$ & $\left\lceil\frac{m q}{p}\right\rceil \cdot \log _{2} p$ \\
\hline
\end{tabular}

Fig. 4. Comparisons for matrix-matrix multiplication algorithms.

\section{3: Redistribution under Multiple Faults}

When some processors are faulty and the errors are detected, the jobs they are performing must be redistributed to other fault-free processors. This is the basic concept of load redistribution. We use the row/column UDD (Uniform Data Distribution) strategy, i.e. the work load of the faulty processors is first redistributed row-wisely and then column-wisely [2]. Two cases of multiple faults are considered in this section - either all faults are in one row or in one column.

Assume there are $r$ faulty processors in a row and totally $p \times p$ processors in the hypercube.

Let: $w$ be the width of the original largest submatrix, $h$ be the height of the original largest submatrix,

$w_{1 f}$ be the width of submatrices distributed after row-wise UDD,

$h_{1,}$ be the reduced height of submatrices in the row with faulty nodes, $h$, be the increased height of submatrices distributed to the remaining rows.

Therefore, $w=\lceil n / p\rceil$ and $h=\lceil m / p\rceil$ in the Simple Algorithm; $w=\lceil n q / p\lceil$ and $h=\lceil m / p\rceil$ in Algorithm CE; $w=\lceil n / p\rceil$ and $h=\lceil m g / p\rceil$ in Algorithm RE. The following equations must hold in order for each node to have an equal number of data elements after the UDD (see Fig. 5(a)): 
$w \cdot h_{1}=w_{1 f} \cdot h_{1 f}$

$h_{1 f}+(p-1) \cdot h_{1}=p \cdot h$

$w_{1 f}=w+r w /(p-r)$.

After a sequence of operations on the above equations, we get:

$h_{1 f}=h p(p-r) /\left(p^{2}-r\right)$ and $w_{1 f}=w p /(p-r)$.

Assume there are $c$ faulty processors in a column, and again, $p \times p$ processors in the hyper-

cube. Similarly, from Fig. S(b), we have:

$w \cdot h_{1}=w_{1 f} \cdot h_{1 f}$

$c \cdot h_{1 f}+(p-c) \cdot h_{1}=p \cdot h$

$w_{1 f}=w+w /(p-1)$.

After manipulating these equations, the final results are:

$h_{1 f}=h p(p-1) /\left(p^{2}-c\right) ; w_{1 f}=w p /(p-1)$.

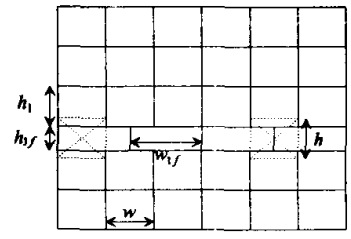

load of a faulty processor

Faults in a row

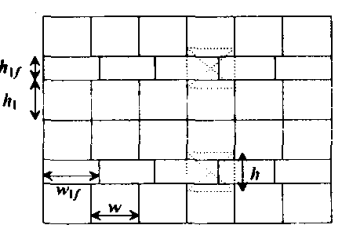

(b) Faults in a column Fig. 5. Matrix partitioning after row/column-UDD.

\section{4: Conclusions}

In this paper, three algorithms for matrix-matrix multiplications are proposed and compared in terms of the number of multiplications, additions, and transfers. Furthermore, faulttolerance issues for these algorithms are also discussed. These algorithms explore the connectivity of the hypercube and therefore are communication efficient. The transfer complexity of these algorithms is $\mathrm{O}\left(\log _{2} p\right)$ if $p \times p$ processors are used. Overall, Algorithm RE behaves better than the other two algorithms with better load balance, and lower communication overhead.

[1] A. C. Elster and A. P. Reeves, "Block-matrix Operations Using Orthogonal Trees," Proceeding of the Third International Conference on Hypercube Multiprocessors, SIAM, Pasadena, CA (January, 1988).

[2] A. C. Elster, M. Umit Uyar, and Anthony P. Reeves, "Fault-Tolerant Matrix Operations on Hypercube Multiprocessors", International Conference on Parallel Processing (1989).

[3] G. H. Golub and C. F. Van Loan, Matrix Computations, Johns Hopkins, Baltimore, MD (1983).

[4] J. Salmon, "Binary Gray Codes and the Mapping of Physical Lattice into a Hypercube," Caltech Concurrent Processor Report (CCP) Hm-51, (1983).

[5] S. L. Johnson, "Communication Efficient Basic Linear Algebra Computations on Hypercube Architecture," Journal of Parallel and Distributed Computing, 4, pp. 133-172 (1987).

[6] O. A. Mcbryan and E. F. Van de Velde, "Matrix and Vector Operations on Hypercube Parallel Processors," Parallel Compuing, 5,(1\&2), pp. 117-125, North-Holland, (July 1987).

17] Angel L. Decegama, The technology of Parallel Processing (Purallel Processing Architecture and VLSI Hardware Volume 1), Prentice Hall International Editions (1989). 